\title{
Effect of tartaric acid and dietary fibre from sun-dried raisins on colonic function and on bile acid and volatile fatty acid excretion in healthy adults
}

\author{
Gene A. Spiller ${ }^{1 *}$, Jon A. Story ${ }^{2}$, Emily J. Furumoto ${ }^{2}$, Jo Carol Chezem ${ }^{2}$ and Monica Spiller ${ }^{1}$ \\ ${ }^{1}$ Sphera Foundation, PO Box 338, Los Altos, California 94023, USA \\ ${ }^{2}$ Department of Foods and Nutrition, 1264 Stone Hall, Purdue University, W. Lafayette, Indiana 47907-1264, USA
}

(Received 17 September 2002 - Revised 8 May 2003 - Accepted 18 June 2003)

\begin{abstract}
Sun-dried raisins are a source of dietary fibre and tartaric acid. The effects of tartaric acid on colon function have not been the focus of extensive research. The purpose of the present study was to evaluate the effects of dietary fibre and tartaric acid from sun-dried raisins on colon function and on faecal bile acid and short-chain fatty acid (SCFA) excretion in healthy adults. Thirteen healthy subjects were fed $120 \mathrm{~g}$ sun-dried raisins/d or $5 \mathrm{~g}$ cream of tartar (equivalent to the tartaric acid in $120 \mathrm{~g}$ sun-dried raisins)/d for 9 weeks, divided into 3-week cycles. The experimental diets were fed in a crossover design after an initial control period. Faeces were collected for the last $4 \mathrm{~d}$ of each cycle for analysis of SCFA and bile acids. Intestinal transit time decreased from $42 \mathrm{~h}$ on the baseline diet to $31 \mathrm{~h}$ on cream of tartar $(P<0 \cdot 1)$ and to $28 \mathrm{~h}$ on sun-dried raisins $(P<0 \cdot 05)$. Faeces were softer on both sun-dried raisins and cream of tartar, but sun-dried raisins increased faecal wet weight $(P<0 \cdot 05)$, while cream of tartar did not. Sun-dried raisins caused significant reductions from baseline values in total bile acid concentration (from 1.42 (SD 1.03) to 1.09 (SD 0.76) $\mathrm{mg} / \mathrm{g}, P<0.05$ ), whereas cream of tartar did not (1.40 (SD 1.06) mg/g). Sun-dried raisins also significantly reduced the lithocholic (LC):deoxylithocholic acid (DC) ratio (from 1.63 (SD 0.85) to 1.09 (SD 0.50), $P<0.02)$, whereas cream of tartar reduced the ratio, but to a lesser extent (1.29 (SD 0.79), NS). Both faecal bile acids and the LC:DC ratio are indicators of reduced risk for colon cancer. Sun-dried raisins increased total SCFA excretion (from 5.6 (SD 3.4) to 7.6 (SD 3.0) g/4 d, $P<0.05$ ), which remained unchanged with cream of tartar (5.6 (SD 3.0) g/4 d). Both sun-dried raisins and cream of tartar appear to be good stool softeners and to shorten intestinal transit time, although the fibre in sun-dried raisins has the added benefit of increasing faecal weight. Both sun-dried raisins and cream of tartar modulate the composition of faecal bile acids and SCFA in a way that has potential health benefits.
\end{abstract}

Sun-dried raisins: Tartaric acid: Gastrointestinal transit time: Faecal bile acids

Sun-dried raisins are a source of both soluble and insoluble fibre $(60-80 \mathrm{~g} / \mathrm{kg}$ raisins $)$ and of tartaric acid, a component present in significant amounts in grapes and raisins in temperate climates and in tamarinds in tropical ones (Table 1). As tartaric acid has low solubility in water, some of its content is lost in wine making and in grape juice, leaving grapes and sun-dried raisins as the most practical sources of tartaric acid.

Dietary fibre is known to improve colonic function by modulating gastrointestinal transit time, faecal weight, faecal acidity, faecal short-chain fatty acids (SCFA) and faecal bile acids (Story \& Furumoto, 1990; Cummings, 2001; Kobayashi \& Fleming, 2001). While dietary fibre has been studied extensively in the past 20 years, and its beneficial effects on colonic function are well established, tartaric acid has not been the focus of extensive research. Unlike other fruit acids (such as malic and citric acids), which are digested and converted to energy in the body, tartaric acid bypasses the small intestine and is fermented by colonic bacteria, which utilize it for the production of SCFA, just as they use dietary fibre. The products of this fermentation may increase the acidity of the faeces, a characteristic observed in populations with low rates of colon cancer (International Agency for Cancer Research, 1997).

A second result of microbial fermentation is an increase in stool mass due to increases in microbial products and gases (Cummings, 2001). Many studies have shown that transit time decreases as faecal weight increases to about $200 \mathrm{~g} / \mathrm{d}$ (Cummings, 2001; Spiller \& Spiller, 2001a). While the ideal transit time for human subjects has not been established, it can be assumed that a shorter transit time is preferable, in that this leads to wetter stools that are easier to eliminate and hastens the elimination of carcinogens (Spiller \& Spiller, 2001a).

The metabolism of bile acids plays a key role in the aetiology of colon cancer. Epidemiological and experimental animal studies have shown that the risk for 
Table 1. Tartaric acid content of common foods*

\begin{tabular}{lc}
\hline Food & Tartaric acid \\
\hline Sun-dried raisins $(\mathrm{g} / \mathrm{kg})$ & $20-35$ \\
Grapes (fresh; $\mathrm{g} / \mathrm{kg})$ & $6-9$ \\
Wine $(\mathrm{g} / \mathrm{l})$ & $5-7$ \\
\hline
\end{tabular}

* Spiller \& Spiller (2001b).

colon cancer increases with increased concentrations of faecal bile acids (Hill, 1991). Dietary fibre has been shown to decrease the concentration of bile acids in the faeces (Hosig et al. 1996). Individual bile acids have long been implicated as promoters of carcinogenesis (Aries et al. 1969) and observational studies have observed a relationship between specific ratios of bile acids, i.e. lithocholic acid (LC):deoxycholic acid (DC), and colorectal cancer risk (Owen et al. 1987). More recent work has reported a more direct in vitro effect of bile acids on cell proliferation as well as apoptosis, suggesting a mechanism for their observed promotional effects in carcinogenesis (Qiao et al. 2000, 2001).

In the present study, the effects of both sun-dried raisins and pure tartaric acid (in the form of cream of tartar) on indicators of colonic function and on SCFA and faecal bile acid excretion have been tested against a control diet free from grape products and low in fibre.

\section{Methods}

\section{Subjects}

Thirteen healthy adults (seven male and six female), 27-65 years of age (47 (SD 13) years) from the San Francisco Bay area (CA, USA) volunteered to participate in the study. The study protocol had been approved by an independent review committee and was explained to each subject, who then signed an informed consent. Participants also signed the subject's Bill of Rights and were told that they were free to withdraw from the study at any time.

\section{Study design}

A randomized, controlled, crossover design was used to compare the effects of sun-dried raisins and cream of tartar over a 9-week period, divided into 3-week cycles. In cycle 1 (baseline period), subjects consumed their normal diet, with no grape products and a limited amount of fibre. In cycle 2 , seven subjects added $120 \mathrm{~g}$ sun-dried raisins (three packets of approximately $42 \mathrm{~g}$ raisins each) and six subjects added $5 \mathrm{~g}$ cream of tartar, to be taken with meals (approximately equivalent to the tartaric acid in $120 \mathrm{~g}$ sun-dried raisins). In cycle 3 , the sun-dried-raisin group switched to cream of tartar and vice versa. Subjects kept a record of frequency of faecal eliminations and ease or difficulty of expulsion. Subjects were also asked to keep $4 \mathrm{~d}$ food records at the beginning of each cycle: these were reviewed by a nutritionist to ensure that fibre intakes were kept constant, with the exception of the fibre added by the sun-dried raisins in cycles 2 and 3.

\section{Measurements}

Stools were collected for the last $4 \mathrm{~d}$ of each cycle (third week) in containers: they were immediately frozen and later X-rayed, weighed and homogenized for future analysis. Faeces were analysed for moisture (\%) and dry weight.

Intestinal transit time was measured according to the method of Hinton et al. (1969). Transit markers, in the form of forty radio-opaque pellets, were administered to the subjects at 09.00 hours of the first day of faecal collections and $80 \%$ appearance of the markers in the faeces was taken as the transit time.

Faecal bile acids analysis was carried out by extraction of lyophilized faeces with methanol-chloroform $(1: 1, \mathrm{v} / \mathrm{v})$ at $65^{\circ} \mathrm{C}$ for $4 \mathrm{~h}$ and deconjugation of any remaining bile salts enzymatically using $6 \mathrm{mg}$ choloylglycine hydrolase with shaking. Bile acids were then extracted with diethyl ether and ethyl acetate after lowering $\mathrm{pH}$ to 1 with $\mathrm{HCl}$ and further purified by elution from a Sep-Pak $\mathrm{C}_{18}$ column (Waters Corp., Milford, MA, USA) with methanol after pre-treating the column with water. Methanol was removed under $\mathrm{N}_{2}$ and bile acids re-dissolved in dry methanol and methylated with concentrated $\mathrm{HCl}$ and dimethoxypropane.

Methylated bile acids were silylated and quantified by GLC using a $30 \mathrm{~m}$ DB1701 capillary column (J \& W Scientific, Folson, CA, USA), using $5 \beta$-cholanic acid as an internal standard (Chezem \& Story, 1997). The LC:DC and chenodeoxycholic acid:cholic acid ratios were computed, and the concentration of bile acids/g faecal weight was calculated.

Faecal SCFA assays were performed by GLC (Agway Inc., Analytical Laboratory, Ithaca, NY, USA). Column temperature was programmed from 80 to $150^{\circ} \mathrm{C}$ at $20^{\circ} \mathrm{C} / \mathrm{min}$. To $1 \mathrm{~g}$ faecal homogenate, $0.1 \mathrm{ml} \mathrm{H}_{3} \mathrm{PO}_{4}$ $(850 \mathrm{~g} / \mathrm{l})$ and a known amount of caprylic acid (internal standard) were added and then extracted three times with anhydrous diethyl ether, centrifuged, and the diethyl ether layer transferred to a $10 \mathrm{ml}$ volumetric flask and diluted to the mark with diethyl ether. A sample of the diethyl ether extract $(3 \mu \mathrm{l})$ was injected directly onto the column. Calibration and calculation were made by the internal standard method. Calibration curves were obtained for acetic, propionic, isobutyric, butyric, isovaleric and valeric acids by chromatography of standard solutions at five different concentrations. The calibration curves were prepared by plotting the area ratios (weight of active compound per weight of internal standard).

\section{Statistical analysis}

Student's paired $t$ tests (two-tailed) were used to compare changes in transit time, daily faecal weight, faecal moisture (\%), dry weight and SCFA. Since only a decrease in faecal bile acid concentrations was of interest, a one-sided $t$ test of statistical significance was used to evaluate changes in total and individual bile acid concentrations and in the LC:DC ratios. In order to reduce the between-subject variation, the baseline values for total bile acids, DC, LC, and the LC:DC ratios for each subject were subtracted from the values at the end of each dietary treatment. Results, presented as mean values and standard deviations, were considered significant at the $P<0.05$ level. 


\section{Results}

\section{Colonic function}

Intestinal transit time decreased from 42 (SD 18) $\mathrm{h}$ for the baseline period to 31 (SD 10) $\mathrm{h}$ for the cream-of-tartar period $(P<0.1)$ and to 28 (SD 11) $\mathrm{h}$ for the sun-dried raisin period $(P<0.05)$ (Fig. 1). The number of radioopaque transit pellets passed by all subjects in the first $24 \mathrm{~h}$ also increased for both the cream-of-tartar and the sun-dried-raisin periods $(P<0.05)$ (Fig. 2). The changes in daily faecal weight are shown in Fig. 3. Daily faecal weight increased slightly for the cream-of-tartar period, from 132 (SD 68) g during baseline to 142 (SD 81) g (NS). During the sun-dried-raisin period, daily faecal weight showed a statistically significant increase to 177 (SD 78) g $(P<0 \cdot 05)$. The moisture in the stools (Fig. 4) increased somewhat during the sun-dried-raisin period (79 (SD 5) \%, $P<0 \cdot 2$ ) compared with baseline (76 (SD 5) \%), but not during the cream-of-tartar period (76 (SD 6) \%). Similarly, DM decreased non-significantly $(P<0 \cdot 2)$ in the sun-driedraisin period (21 (SD 5) \%) compared with baseline $(24$ (SD 5) \%), but not in the cream-of-tartar period (24 (SD 6) \%). The subjects' records of bowel function showed that stools were softer and easier to eliminate in both sundried-raisin and cream-of-tartar periods.

\section{Bile acids and short-chain fatty acids}

The results of bile acids analyses are shown in Table 2 . Daily excretion of faecal bile acids was not significantly altered by either dietary treatment. However, consumption of sun-dried raisins did reduce the concentration of total bile acids in the faeces $(P<0.05)$, whereas treatment with cream of tartar did not. The concentration of LC was

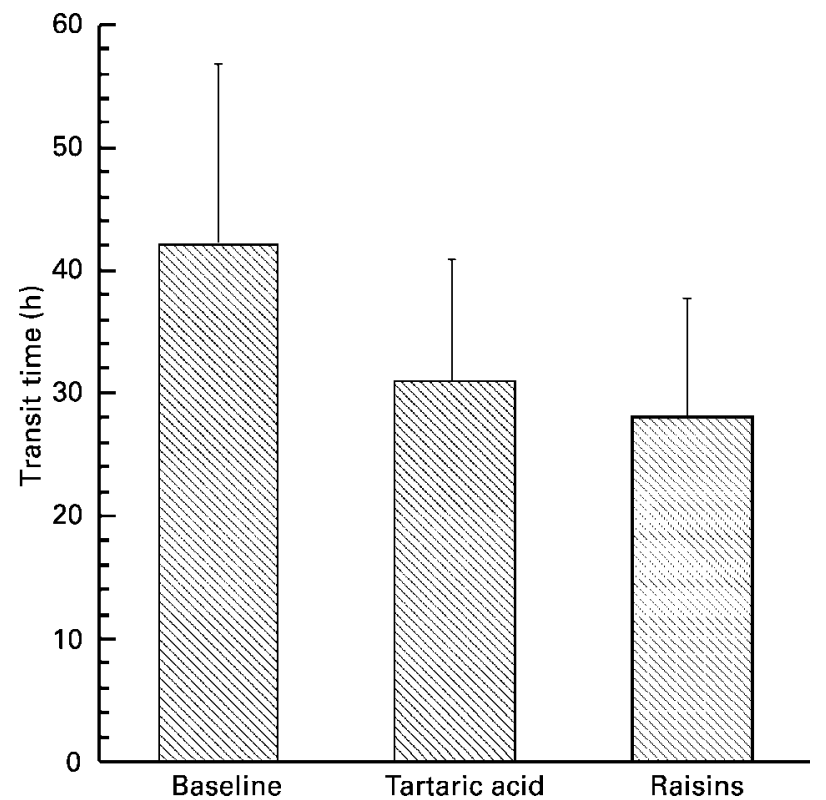

Fig. 1. Effect of sun-dried raisins $(120 \mathrm{~g} / \mathrm{d}$ for 3 weeks) or tartaric acid ( $5 \mathrm{~g}$ cream of tartar/d for 3 weeks) on gastrointestinal transit time (time (h) to excrete $80 \%$ of radio-opaque pellets). Values are means with standard deviations shown by vertical bars (thirteen per group). For details of subjects and procedures, see p. 804. significantly reduced by sun-dried raisins $(P<0.005)$, as was the LC:DC ratio $(P<0.02)$, while the concentration of DC was reduced (NS). No change was observed in the individual bile acids in response to cream-of-tartar feeding, but the LC:DC ratio was reduced $(P<0 \cdot 08)$.

The results of the SCFA analyses are shown in Table 3. Sun-dried raisins resulted in a significant increase in total faecal SCFA and in some individual SCFA (acetic, propionic, butyric and isovaleric; $P<0.05)$.

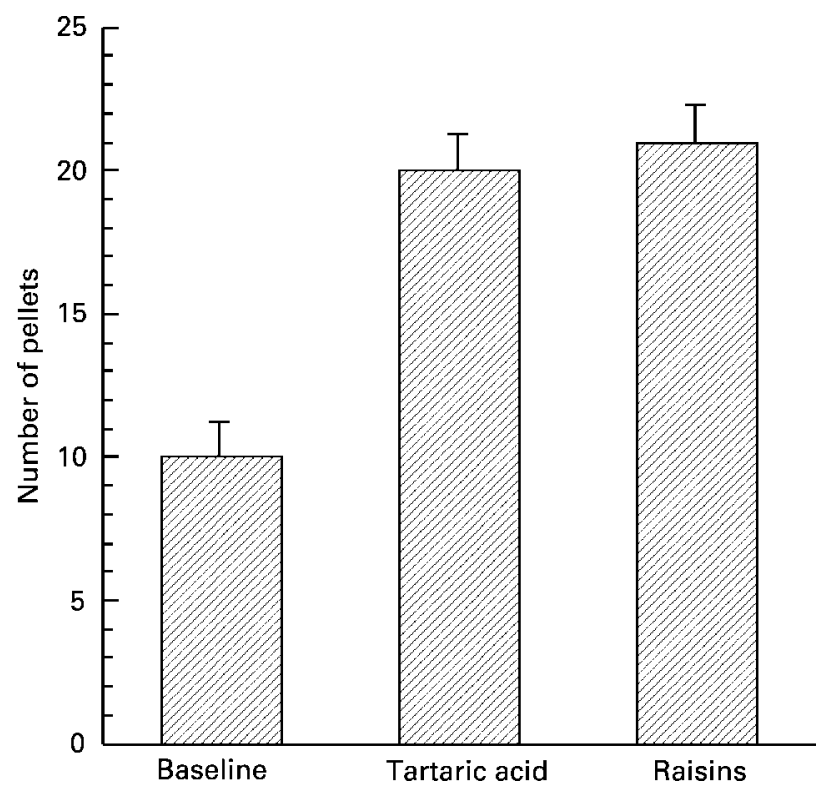

Fig. 2. Number of radio-opaque pellets excreted in the first $24 \mathrm{~h}$ of each 3-week treatment period (sun-dried raisins $(120 \mathrm{~g} / \mathrm{d})$ or tartaric acid $(5 \mathrm{~g}$ cream of tartar $/ \mathrm{d})$ ). Values are means with standard deviations shown by vertical bars (thirteen per group). For details of subjects and procedures, see p. 804 .

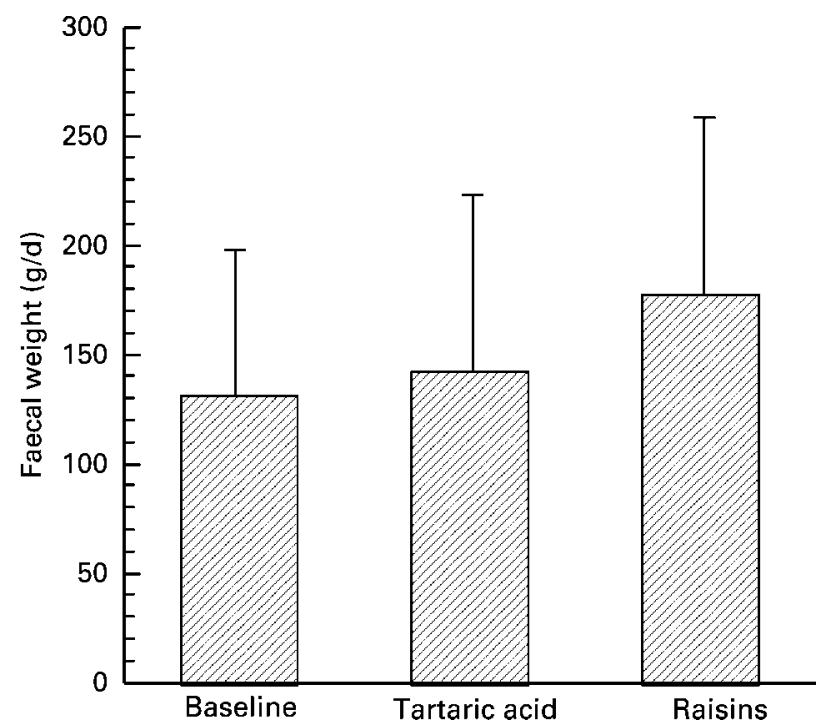

Fig. 3. Effect of sun-dried raisins $(120 \mathrm{~g} / \mathrm{d}$ for 3 weeks) or tartaric acid ( $5 \mathrm{~g}$ cream of tartar/d for 3 weeks) on faecal weight. Values are means with standard deviations shown by vertical bars (thirteen per group). For details of subjects and procedures, see p. 804. 


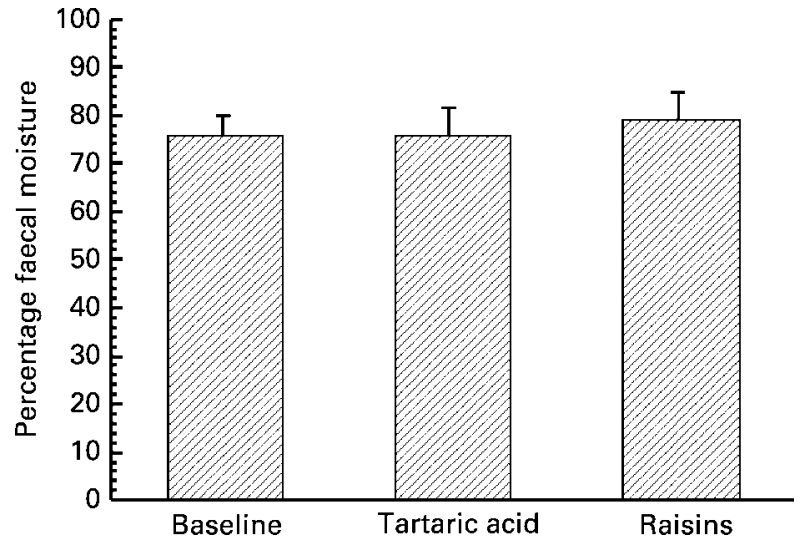

Fig. 4. Effect of sun-dried raisins $(120 \mathrm{~g} / \mathrm{d}$ for 3 weeks) or tartaric acid ( $5 \mathrm{~g}$ cream of tartar $/ \mathrm{d}$ for 3 weeks) on faecal moisture (\%). Values are means with standard deviations shown by vertical bars (thirteen per group). For details of subjects and procedures, see p. 804.

\section{Discussion}

In the present study, we tested the effects of feeding $120 \mathrm{~g}$ sun-dried raisins or tartaric acid (as cream of tartar) in an amount equivalent to that present in the sun-dried raisins, on indicators of colonic function, as well as on the excretion of faecal bile acids and SCFA, in healthy adults. The results of the present study suggest that both sun-dried raisins and cream of tartar are good stool softeners, but the fibre of sun-dried raisins has the added benefit of increasing faecal weight. It is known that an inverse correlation exists between transit time and faecal weight up to $200 \mathrm{~g} / \mathrm{d}$ (Cummings, 2001; Spiller \& Spiller, 2001a) and our current results confirm this. During the sun-dried-raisin cycle, faecal weight increased significantly $(+45 \mathrm{~g} / \mathrm{d})$ to about $180 \mathrm{~g} / \mathrm{d}$; subjects experienced a significant reduction in transit time $(-14 \mathrm{~h})$ compared with the cycle in which subjects were on their normal low-fibre diet. Although during the cream-of-tartar cycle the changes were not statistically significant, stool weight increased somewhat $(+10 \mathrm{~g} / \mathrm{d})$ and transit time showed a $9 \mathrm{~h}$ reduction compared with the baseline diet period, suggesting an additive effect of the tartaric acid and the fibre. Mechanisms by which these changes in transit time occur are suggested by some of the remaining results. The increase in $\%$ moisture in response to sun-dried raisins $(+3 \%)$ would soften stools and increase the rate of passage through the intestine. However, this change was not statistically significant and no change in moisture was observed in response to cream of tartar, but the reduction in transit time was similar to that observed in response to sun-dried raisins. Faecal SCFA have been suggested to soften stools by acting as an osmotic agent, retaining water in faeces. Not surprisingly, since $\%$ moisture was not increased by either treatment,

Table 2. Faecal bile acids for the baseline, cream-of-tartar and sun-dried-raisin periods $\dagger$ (Mean values and standard deviations for thirteen subjects)

\begin{tabular}{|c|c|c|c|c|c|c|}
\hline & \multicolumn{2}{|c|}{ Baseline } & \multicolumn{2}{|c|}{ Cream of tartar } & \multicolumn{2}{|c|}{ Sun-dried raisins } \\
\hline & Mean & SD & Mean & SD & Mean & SD \\
\hline Bile acid excretion (mg/d) & 187 & 135 & 200 & 151 & 194 & 134 \\
\hline \multicolumn{7}{|l|}{ Bile acid concentrations (mg/g) } \\
\hline Total & 1.42 & 1.03 & 1.40 & 1.06 & $1.09^{*}$ & 0.76 \\
\hline Deoxycholic acid & 0.55 & 0.67 & 0.58 & 0.62 & 0.45 & 0.43 \\
\hline Lithocholic acid & 0.70 & 0.36 & 0.61 & 0.33 & $0.45^{\star * *}$ & 0.27 \\
\hline Cholic acid & 0.08 & 0.18 & 0.11 & 0.26 & 0.10 & 0.19 \\
\hline Chenodeoxycholic acid & 0.05 & 0.09 & 0.07 & 0.11 & 0.08 & 0.09 \\
\hline Ursodeoxycholic acid & 0.04 & 0.09 & 0.02 & 0.05 & 0.05 & $0 \cdot 10$ \\
\hline Lithocholic: deoxycholic acid ratioł & 1.63 & 0.85 & $1 \cdot 29$ & 0.79 & $1 \cdot 09^{\star *}$ & 0.50 \\
\hline
\end{tabular}

Mean values were significantly different from those at baseline: ${ }^{\star} P<0.05,{ }^{\star \star} P<0.02,{ }^{\star \star \star} P<0.005$.

$\dagger$ For details of subjects and procedures, see p. 804

$\ddagger n$ 11: results from two subjects were excluded (outliers).

Table 3. Faecal short-chain fatty acids (SCFA) for the baseline, cream-of-tartar and sun-dried-raisin periodst (Mean values and standard deviations for thirteen subjects)

\begin{tabular}{|c|c|c|c|c|c|c|}
\hline & \multicolumn{2}{|c|}{ Baseline } & \multicolumn{2}{|c|}{$\begin{array}{c}\text { Cream of } \\
\text { tartar }\end{array}$} & \multicolumn{2}{|c|}{$\begin{array}{l}\text { Sun-dried } \\
\text { raisins }\end{array}$} \\
\hline & Mean & SD & Mean & SD & Mean & SD \\
\hline Total SCFA (g/4 d) & $5 \cdot 6$ & $3 \cdot 4$ & $5 \cdot 6$ & $3 \cdot 0$ & $7 \cdot 6^{\star}$ & $3 \cdot 0$ \\
\hline Acetic acid $(g / 4 d)$ & $2 \cdot 8$ & $1 \cdot 8$ & 2.9 & $1 \cdot 6$ & $4 \cdot 0^{*}$ & $1 \cdot 7$ \\
\hline Propionic acid (g/4 d) & $1 \cdot 1$ & 0.6 & $1 \cdot 1$ & 0.6 & $1.4^{\star}$ & 0.6 \\
\hline Isobutyric acid (g/4 d) & 0.13 & 0.05 & 0.13 & 0.04 & 0.15 & 0.04 \\
\hline Butyric acid $(g / 4 d)$ & $2 \cdot 3$ & 2.7 & $2 \cdot 2$ & $2 \cdot 6$ & $2 \cdot 7^{* *}$ & 3.6 \\
\hline Isovaleric acid (g/4 d) & 0.12 & 0.04 & 0.12 & 0.07 & $0 \cdot 16^{*}$ & 0.05 \\
\hline Valeric acid $(g / 4 d)$ & 0.21 & 0.16 & 0.15 & 0.07 & 0.19 & 0.08 \\
\hline
\end{tabular}

Mean values were significantly different from those at baseline: ${ }^{*} P<0.05,{ }^{*} P<0.02$.

†For details of subjects and procedures, see p. 804 . 
concentrations of SCFA were not changed (control $10.6 \mathrm{mg} / \mathrm{g}$, tartaric acid $9.8 \mathrm{mg} / \mathrm{g}$, sun-dried raisins $10.7 \mathrm{mg} / \mathrm{g}$ ) in spite of an increase in daily output of SCFA in the sun-dried-raisin group. The softening of stools and improved ease of elimination reported by subjects in response to both sun-dried-raisin and cream-oftartar treatments does not seem to be a result of any of these common mechanisms. Changes in the microbial population of the intestine in response to tartaric acid and/or the dietary fibre components of sun-dried raisins could cause the increase in faecal weight and reduction in transit time (Stephen \& Cummings, 1980).

Faecal bile acid concentration has been directly associated with colo-rectal cancer risk for some time (Hill, 1991). Early observations that DC and LC acted as co-mutagenic compounds in a variety of in vitro mutagenicity assays supported this concept (Wilpart et al. 1983; Wilpart \& Roberfroid, 1986). A more specific role of these bile acids in the carcinogenesis process has been elucidated in recent years. Studies in human colon cancer cell lines have indicated that bile acids act as promoters of carcinogenesis by their action on a number of signalling pathways that alter both cell proliferation and apoptosis (Martinez et al. 1998; Schlottmann et al. 2000; Qiao et al. 2000, 2001). These results reporting a reduction in both the concentrations of DC (although not statistically significant) and LC indicate a positive response in terms of reduction of a potential promoter of carcinogenesis. Mechanistic studies in human subjects are needed to examine and substantiate these relationships.

Epidemiological observations have suggested that the $\mathrm{LC}: \mathrm{DC}$ ratio is more predictive of colo-rectal cancer risk than total bile acid concentration or individual bile acids alone (Owen et al. 1987). This hypothesis has been further supported by mutagenicity testing (Qiao et al. 2000), although it has not been examined in the more specific systems discussed earlier. In the present study, the LC:DC ratio was reduced to about 1.00 in response to consumption of sun-dried raisins, and reduced somewhat by cream-oftartar consumption, indicating a reduced risk for development of colo-rectal cancer. Again, further mechanistic examination of this relationship is needed to understand the specific nature of bile acid promotion of carcinogenesis and the role diet can play in reducing this risk.

In conclusion, the results of the present investigation suggest that sun-dried raisins have beneficial effects on intestinal function that appear to be mediated by both its dietary fibre and tartaric acid contents. In addition, sun-dried raisins and tartaric acid appear to have potential benefits in decreasing the risk for colo-rectal cancer. These beneficial effects may be one of the reasons why grapes and raisins have been considered healthy foods for millennia.

\section{Acknowledgements}

We wish to acknowledge Judy Silva, RN and Alexandra Glezerman, RN from HCR Manage Care (Sunnyvale, CA, USA) for their assistance, Rex McIntosh for his laboratory work and Antonella Dewell, MS, RD for her work on the manuscript. The study was supported by an unrestricted grant by Sun-Maid Growers of California.

\section{References}

Aries VC, Crowther JS, Drasar BS \& Hill MJ (1969) Degradation of bile salts by human intestinal bacteria. Gut 10, 575-577.

Chezem JC \& Story JA (1997) Development of an updated method for fecal bile acid and neutral steroid analysis. Am Clin Lab 16, 20-21.

Cummings JH (2001) The effect of dietary fiber on fecal weight and composition. In Dietary Fiber in Human Nutrition, pp. 183-252 [GA Spiller, editor]. Boca Raton, FL: CRC Press.

Hinton JM, Lennard-Jones JE \& Young AC (1969) A new method of studying gut transit times using radio-opaque markers. Gut 10, 842 .

Hill MJ (1991) Bile acids and colorectal cancer: Hypothesis. Eur J Cancer Prev 1, Suppl. 2, 69-72.

Hosig KW, Shinnick FL, Johnson MD, Story JA \& Marlett JA (1996) Comparison of large bowel function and calcium balance during soft wheat bran and oat bran consumption. Cereal Chem 73, 392-398.

International Agency for Cancer Research (1977) Dietary fiber, transit time, fecal bacteria and colon cancer in two Scandinavian populations. Lancet i, 207.

Kobayashi H \& Fleming SE (2001) The source of dietary fiber influences. Short-chain fatty acid production and concentrations in the large bowel. In Dietary Fiber in Human Nutrition, pp. 287-316 [GA Spiller, editor]. Boca Raton, FL: CRC Press.

Martinez JD, Stratagoules ED, LaRue JM, et al. (1998) Different bile acids exhibit distinct biological effects: the tumor promoter deoxycholic acid induces apoptosis and the chemopreventive agent ursodeoxycholic acid inhibits cell proliferation. Nutr Cancer 31, 111-118.

Owen RW, Thompson MH, Hill MJ, Wilpart M, Mainguet P \& Roberfroid M (1987) The importance of the ratio of lithocholic to deoxycholic acid in large bowel carcinogenesis. Nutr Cancer 9, 67-71.

Qiao D, Chen W, Stratagoules ED \& Martinez JD (2000) Bile acidinduced activation of activator protein-1 requires both extracellular signal-regulated kinase and protein kinase $\mathrm{C}$ signaling. J Biol Chem 275, 15090-15098.

Qiao D, Gaitonde SV, Qi W \& Martinez JD (2001) Deoxycholic acid suppresses $\mathrm{p} 53$ by stimulating proteasome-mediated p53 protein degradation. Carcinogenesis 22, 957-964.

Schlottmann K, Wachs F-P, Krieg RC, Kullmann F, Schölmerich J \& Rogler G (2000) Characterization of bile salt-induced apoptosis in colon cancer cell lines. Cancer Res 60, 4270-4276.

Spiller GA \& Spiller M (2001a) Correlations of transit time to a critical fecal weight (CFW) and to substances associated with dietary fiber. In Dietary Fiber in Human Nutrition, pp. 253-256 [GA Spiller, editor]. Boca Raton, FL: CRC Press.

Spiller GA \& Spiller M (2001b) Tartaric acid content of foods. In Dietary Fiber in Human Nutrition, p. 681 [GA Spiller, editor]. Boca Raton, FL: CRC Press.

Stephen AM \& Cummings JH (1980) Mechanisms of actions of dietary fibre in the human colon. Nature 284, 283-284.

Story JA \& Furumoto EJ (1990) Dietary fiber and bile acid metabolism. In Dietary Fiber, pp. 365-374 [D Kritchevsky, C Bonfield and JW Anderson, editors]. New York: Plenum.

Wilpart M, Mainguet P, Makens A \& Roberfroid M (1983) Structure-activity relationships among bile acids showing comutagenic activity towards DMH. Carcinogenesis 4, $1239-1241$

Wilpart M \& Roberfroid M (1986) Effects of secondary bile acids on the mutagenicity of MNNG, 2AAF and 2-nitrofluorene towards Salmonella typhimurium strains. Carcinogenesis 7, 703-706. 